\title{
Satellite Attitude Control System Design considering the Fuel Slosh Dynamics
}

\author{
Luiz Carlos Gadelha de Souza and Alain G. de Souza \\ National Institute for Space Research (INPE/DMC), Avenida dos Astronautas 1758, 12227-010 São José dos Campos, SP, Brazil \\ Correspondence should be addressed to Luiz Carlos Gadelha de Souza; gadelha@dem.inpe.br
}

Received 26 June 2013; Accepted 8 January 2014; Published 26 June 2014

Academic Editor: Nuno Maia

Copyright ( 2014 L. C. G. de Souza and A. G. de Souza. This is an open access article distributed under the Creative Commons Attribution License, which permits unrestricted use, distribution, and reproduction in any medium, provided the original work is properly cited.

\begin{abstract}
The design of the satellite attitude control system (ACS) becomes more complex when the satellite structure has different type of components like, flexible solar panels, antennas, mechanical manipulators, and tanks with fuel. A crucial interaction can occur between the fuel slosh motion and the satellite rigid motion during translational and/or rotational manoeuvre since these interactions can change the satellite centre of mass position damaging the ACS pointing accuracy. Although, a well-designed controller can suppress such disturbances quickly, the controller error pointing may be limited by the minimum time necessary to suppress such disturbances thus affecting the satellite attitude acquisition. As a result, the design of the satellite controller needs to explore the limits between the conflicting requirements of performance and robustness. This paper investigates the effects of the interaction between the liquid motion (slosh) and the satellite dynamics in order to predict what the damage to the controller performance and robustness is. The fuel slosh dynamics is modelled by a pendulum which parameters are identified using the Kalman filter technique. This information is used to design the satellite controller by the linear quadratic regulator (LQR) and linear quadratic Gaussian (LQG) methods to perform a planar manoeuvre assuming thrusters are actuators.
\end{abstract}

\section{Introduction}

The problem of interaction between fluid and structure is important when one needs to study the dynamic behavior of offshore and marine structures and road and railroad containers partially filled with a fluid $[1,2]$. In space missions the sloshing problem appears when the spacecraft is spinning and there is liquid inside it. An example is the damp device involving fluid as the damping material, whose motion can interact with flexible panels and/or manipulators [3]. An interesting approach to analyze a rigid container mounted on flexible springs interacting with a perfect fluid including sloshing effects has been done by Lui and Lou [4]. The success of a space mission can depend on taking into account the knowledge of the interaction between fluid motion (slosh) and structure dynamics since this interaction can damage the ACS pointing requirements. A space structure, like rockets, geosynchronous satellites, and the space station, usually contains liquid in tanks that can represent more than $40 \%$ of the initial mass of the system. As a result, the first step to design its ACS is to obtain a detailed dynamics model of the space structure. When the fuel tanks are only partially filled and suffer a transverse acceleration and/or rotational motion, large quantities of fuel move uncontrollably inside the tanks and generate the sloshing effects. Agrawal [5] has shown that the dynamics interaction between the fuel motion and the rigid and/or flexible body dynamics can result in some kind of control instability. For minimizing these effects the ACS must be designed using a robust control method in order to assure stability and good performance to achieve the attitude control system requirement [6]. When a rigid-flexible satellite with fuel tanks inside is subjected to large angle manoeuvre its dynamics is only captured by complex nonlinear mathematical model. Besides, the remaining flexible and/or liquid vibration can introduce a tracking error resulting in a minimum attitude acquisition time. Souza [7] has done a detailed investigation of the influence of the nonlinearities introduced by the panel's flexibility into the ACS design. It was shown that system parameters variation can degrade the control system performance, indicating the necessity to improve 
the ACS robustness. An experimental controller robustness and performance investigation was done by Conti and Souza [8], where the estimation of the platform inertia parameters was introduced as part of the platform ACS design. The problem of designing satellite nonlinear controller for rigid satellite has been done by Souza and Gonzales [9] using the state-dependent Riccati equation (SDRE) method which is able to deal with high nonlinear plants. Due to the complexity of modeling the fluid and/or flexible dynamic of the system it is common to use mechanical systems analogies that describe this dynamic. Besides, if one needs to know some physical parameters related with the slosh or the flexibility dynamics it is common to obtain them by experimental apparatus or some kind of estimating method such as Kalman filter [10].

\section{Satellite with Sloshing Model}

The phenomenon of sloshing is due to the movement of a free surface of a liquid that partially fills a compartment and this movement is oscillating. It depends on the shape of the tank, the acceleration of the gravity, and on the axial/rotational acceleration of the tank. As a representative of the behavior of the total weight of the system it is accepted that when the mass of the liquid oscillates the mass center of the rigid body also oscillates, thereby disturbing the rigid-flexible part of the vehicle under consideration. As an oscillating movement it is natural to consider the wave generated by the movement of the liquid as a stationary wave in all oscillation modes. Each mode of oscillation has a special feature of this phenomenon under study and one observes, in a quantitative sense, how much mass is displaced. Among all the modes that cause the greatest disruption in the system are the first and the second modes. Despite the fact that oscillation has lower frequency it is capable of resulting in violent shifting of the center of mass of the liquid creating an oscillation in the system as a role. The other oscillation modes act as a less aggressive and it may not even vary the position of its center of mass due to the symmetry of the wave which on average causes no displacement. Due to its complexity, the sloshing dynamics is usually represented by mechanical equivalents that describe and reproduce faithfully the actions and reactions due to forces and torques acting on the system. The main advantage of replacing the fluid model with an equivalent oscillating model [11] is simplifying the analysis of motion in the rigid body dynamics compared to the fluid dynamics equations. Due to the complexity of establishing an analytical model for the fluid moving freely within a closed tank, a simplified system is used, taking into account the following criteria [6]: (a) small displacements, (b) a rigid tank, and (c) no viscous, incompressible, and homogeneous liquid. Under these conditions the dynamics of the sloshing can be approximated by mechanical system consisting of a mass-spring or pendulum. Consider a rigid spacecraft moving in a fixed plane with a spherical fuel tank and including the lowest frequency slosh mode. Based on the Lagrange equation and the Rayleigh dissipation function one can model systems using the mechanical mass-spring and pendulum type system, respectively. Figure 1 shows a satellite model where slosh dynamics is represented by its pendulum analogous mechanical system, where the mass of the satellite and the moment of inertia, regardless of the fuel, are given by $m$ and $I$, respectively, and the mass equivalent of fuel and its inertia moment are given by $M_{f}$ and $I_{f}$, respectively. The attitude control of the spacecraft is done by the force $f$ and by the pitch moment $M$. The constant thrust $F$ is responsible for the orbital transfer of the spacecraft with respect to the inertial reference system $(x, y, z)$ and it acts on the center of mass of spacecraft in the longitudinal axis. Also it is given the velocity of the center of the fuel tank $v_{x}, v_{z}$ and the attitude angle $\theta$ of the spacecraft with respect to a fixed reference $(X, Y, Z)$. Besides, $V$ represents the linear velocity and $\omega$ represents the angular velocity of the rigid body. The length of the pendulum is $a$, the distance from satellite center of mass to the pendulum connected point is $b$, and the angle of the pendulum with respect to the spacecraft longitudinal axis is $\psi$, which is assumed in the equilibrium position $(\psi=0)$ about the reference axis $X$.

\section{The Satellite Equations of Motion}

The satellite equations of motion can be derived using the Lagrange equations [10] given by

$$
\begin{gathered}
\frac{\mathrm{d}}{\mathrm{dt}}\left(\frac{\partial L}{\partial V}\right)+\omega^{\times} \frac{\partial L}{\partial V}=\tau_{t}, \\
\frac{\mathrm{d}}{\mathrm{dt}}\left(\frac{\partial L}{\partial \omega}\right)+\omega^{\times} \frac{\partial L}{\partial \omega}+V^{\times} \frac{\partial L}{\partial V}=\tau_{r}, \\
\frac{\mathrm{d}}{\mathrm{dt}}\left(\frac{\partial L}{\partial \dot{\psi}}\right)-\frac{\partial L}{\partial \psi}+\frac{\partial R}{\partial \dot{\psi}}=0 .
\end{gathered}
$$

Details of the equations of motion derivation can be found in [12], where $L$ is the Lagrangian of the system, the generalized coordinates are $V$ and $\omega, R$ is the Rayleigh dissipation function, $\tau_{r}$ is the internal torque, and $\tau_{t}$ is the external torque. Assume that $R, \tau_{r}, \tau_{t}, \omega, V$ are given by

$$
\begin{gathered}
R=\frac{1}{2} \varepsilon \dot{\psi}^{2} ; \quad V=\left[\begin{array}{c}
v_{x} \\
0 \\
v_{z}
\end{array}\right] ; \quad \omega=\left[\begin{array}{l}
0 \\
\dot{\theta} \\
0
\end{array}\right] ; \\
\tau_{t}=\left[\begin{array}{c}
F \\
0 \\
f
\end{array}\right] ; \quad \tau_{r}=\left[\begin{array}{c}
0 \\
M+f b \\
0
\end{array}\right] .
\end{gathered}
$$

The position vector of the satellite mass center with respect to the inertial reference system $(x, y, z)$ (see Figure 1 ) is

$$
\vec{r}=(x-b) i+z \widehat{k} \text {. }
$$

Assuming the relations $v_{x}=\dot{x}+z \dot{\theta}$ and $v_{z}=\dot{z}-x \dot{\theta}$ the satellite velocity is given by

$$
\dot{\vec{r}}=v_{x} \hat{i}+\left(v_{z}+b \dot{\theta}\right) \hat{k}
$$

The position of the mass of fuel with respect to the inertial reference system $(x, y, z)$ (see Figure 1$)$ is given by

$$
\vec{r}_{f}=(x-a \cos (\psi)) \hat{i}+(z+a \sin (\psi)) \widehat{k} .
$$




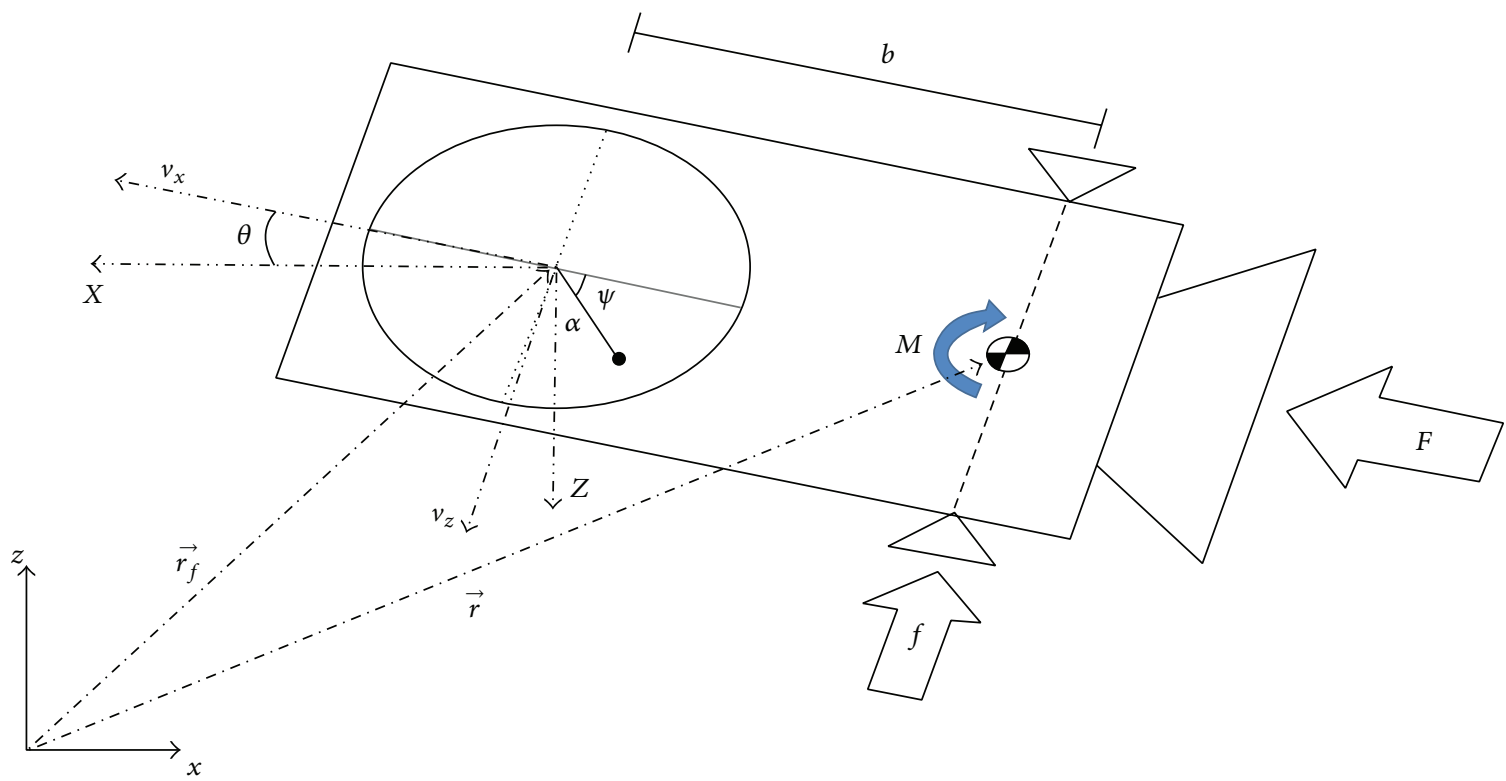

FIGURE 1: Satellite model with slosh dynamics pendulum analogous mechanical system.

As a result, the mass of the fuel velocity is

$$
\begin{aligned}
\dot{\vec{r}}_{f}= & \left(v_{x}+a \sin (\psi)(\dot{\theta}+\dot{\psi})\right) \hat{i} \\
& +\left(v_{z}+a \cos (\psi)(\dot{\theta}+\dot{\psi})\right) \hat{k} .
\end{aligned}
$$

The Lagrangian of the entire system is given by

$$
L=\frac{1}{2} m \dot{\vec{r}}^{2}+\frac{1}{2} m_{f} \dot{\vec{r}}_{f}^{2}+\frac{1}{2} I_{f}(\dot{\theta}+\dot{\psi})^{2}+\frac{1}{2} I \dot{\theta}^{2} .
$$

Substituting (4) and (6) into (7), using the relations given by (2), and performing the derivations of (1), one obtains the satellite equations of motion given by

$$
\begin{gathered}
\left(m+m_{f}\right)\left(\dot{v}_{x}+v_{z} \dot{\theta}\right)+m b \dot{\theta}+m_{f} a(\ddot{\psi}+\ddot{\theta}) \sin (\psi) \\
+m_{f} a(\dot{\theta}+\dot{\psi})^{2} \cos (\psi)=F, \\
\left(m+m_{f}\right)\left(\dot{v}_{z}-v_{x} \dot{\theta}\right)+m_{f} a(\ddot{\theta}+\ddot{\psi}) \cos (\psi) \\
\quad-m_{f} a(\dot{\theta}+\dot{\psi})^{2} \sin (\psi) m b \ddot{\theta}=f, \\
\left(I_{f}+m^{2}\right) \ddot{\theta}+m b\left(\dot{v}_{z}-v_{x} \dot{\theta}\right)-\varepsilon \dot{\psi}=M+b f, \\
\left(m_{f} a^{2}+I_{f}\right)(\ddot{\theta}+\ddot{\psi}) \\
+m_{f} a\left(\left(\dot{v}_{x}+v_{z} \dot{\theta}\right) \sin (\psi)\right. \\
\left.+\left(\dot{v}_{z}-v_{x} \dot{\theta}\right) \cos (\psi)\right)+\varepsilon \dot{\psi}=0 .
\end{gathered}
$$
$m_{f} /\left(m+m_{f}\right)$.

Assuming the relations $a_{x}=\dot{v}_{x}+v_{z} \dot{\theta}, a_{z}=\dot{v}_{z}-v_{x} \dot{\theta}$ and substituting them into (8) and (9), one can isolate and obtain the satellite accelerations given by

$$
\begin{aligned}
& a_{x} \\
& =\frac{F-m b \dot{\theta}-m_{f} a(\ddot{\psi}+\ddot{\theta}) \sin (\psi)-m_{f} a(\dot{\theta}+\dot{\psi})^{2} \cos (\psi)}{m+m_{f}},
\end{aligned}
$$

$a_{z}$

$$
=\frac{f-m_{f} a(\ddot{\theta}+\ddot{\psi}) \cos (\psi)+m_{f} a(\dot{\theta}+\dot{\psi})^{2} \sin (\psi)-m b \ddot{\theta}}{m+m_{f}} .
$$

All equations derived previously are nonlinear. However, in order to design LQR and LQG controllers and estimate the sloshing parameters using the Kaman filter technique one has to get the linear set of equations of motion, which is obtained assuming that the system makes small movements around the point of equilibrium very close to zero; that is, $(\theta, \dot{\theta}, \psi, \dot{\psi})=$ $(0,0,0,0)$.

Now, substituting (12) into (10) and (11) and assuming the linearization conditions, one has the satellite equation of motion given by

$$
\begin{gathered}
\ddot{\theta}\left(I_{f}+m^{*}\left(a^{2}-b a\right)\right)+\psi\left(I_{f}+m^{*} a^{2}\right) \\
+a m_{f}^{*} F \psi+\varepsilon \dot{\psi}=-a m_{f}^{*} f, \\
\ddot{\theta}\left(I+m^{*}\left(b^{2}-b a\right)\right)-m^{*} a b \psi-\varepsilon \dot{\psi}=M+b^{*} f,
\end{gathered}
$$

where $b^{*}=b m_{f} /\left(m+m_{f}\right), m^{*}=m m_{f} /\left(m+m_{f}\right)$, and $m_{f}^{*}=$ 


\section{Kalman Filter (KF)}

The Kalman filter estimates the instantaneous state of a dynamic system from the reading of measurements. In other words the Kalman filter is the optimal solution of minimum variance, which means that the equations of the Kalman filter can be deduced from this premise [7]. The Kalman filtering can be divided into two steps: time update and measurement update.

4.1. Time Update. This step propagates the states and the covariance of the time $t_{k-1}$ to $t_{k}$. For this it just integrates the following equations, with the boundary conditions $\overline{\mathbf{x}}_{k-1}=$ $\widehat{\mathbf{x}}_{k-1}$ and $\overline{\mathbf{P}}_{k-1}=\widehat{\mathbf{P}}_{k-1}$. Herein $\mathbf{P}$ is the covariance matrix and $\mathbf{X}$ is the vector state:

$$
\dot{\overline{\mathbf{x}}}=f(\overline{\mathbf{x}})
$$

where $f$ is a nonlinear vector function of the state $\mathbf{x}$ and time $f$. Consider

$$
\dot{\overline{\mathbf{P}}}=\mathbf{F} \overline{\mathbf{P}}+\overline{\mathbf{P}} \mathbf{F}^{t}+\mathbf{G Q G}^{t}
$$

where $\mathbf{G}$ is a matrix that adds noise to the system dynamic, $\mathbf{Q}$ is the process noise covariance, and $\mathbf{F}$ is the Jacobian matrix of $f$.

4.2. Measurement Update. This step updates the state and covariance to the time $t$ due to the measurement $\mathbf{y}$. Consider the following:

$$
\begin{gathered}
\mathbf{K}_{k}=\overline{\mathbf{P}}_{k} \mathbf{H}_{k}^{t}\left(\mathbf{H}_{k} \overline{\mathbf{P}}_{k} \mathbf{H}_{k}^{t}+\mathbf{R}_{k}\right)^{-1}, \\
\widehat{\mathbf{P}}_{k}=\left(\mathbf{I}-\mathbf{K}_{k} \mathbf{H}_{k}\right) \overline{\mathbf{P}}_{k}, \\
\widehat{\mathbf{x}}_{k}=\overline{\mathbf{x}}_{k}+\mathbf{K}_{k}\left[\mathbf{y}_{k}-h_{k}\left(\overline{\mathbf{x}}_{k}\right)\right],
\end{gathered}
$$

where $K_{k}$ is the filter gain, $H_{k}$ is the Jacobian matrix of $h$ which in turn is a vectorial nonlinear function of the state, and $\mathbf{y}$ is the measurement vector.

\section{Linear Quadratic Gaussian (LQG)}

This method is, basically, the union with the LQR [13] and the Kalman filter. In the LQR method one assumes that all states are available to be feedback but in reality that is not true. Therefore, when there is any state that is not available one has to use the Kalman filter to estimate it in order to be a feedback. The separation principle ensures that each step of this process can be made independently of each other; one may first solve the LQR problem and then design the optimal estimator (Kalman filter), or vice versa, so that the global solution is always the same.

Assume a plant described by the linear state equations given by

$$
\begin{gathered}
\dot{\mathbf{x}}(t)=\mathbf{A} \mathbf{x}(t)+\mathbf{B} \mathbf{u}(t)+\Gamma w, \\
\mathbf{y}=\mathbf{C} \mathbf{x}(t)+v
\end{gathered}
$$

where $\mathbf{x}$ represents the state vector, the matrix $\mathbf{A}$ is the state matrix, $\mathbf{B}$ is the input matrix, $\mathbf{y}$ is the output vector, $\mathbf{C}$ is the output matrix, $v$ and $w$ are white noise, and $\mathbf{u}$ is the control law. In the LQG method [14] the control law gain is obtained by the LQR method and it is given by

$$
\mathbf{K}_{c}=\mathbf{R}^{-1} \mathbf{B}^{T} \mathbf{P}_{c}
$$

where $\mathbf{R}$ is real symmetric positive definite matrix and $\mathbf{P}_{c}$ is the symmetrical solution of the LQR Riccati equation given by

$$
\mathbf{A}^{T} \mathbf{P}_{c}+\mathbf{P}_{c} \mathbf{A}-\mathbf{P}_{c} \mathbf{B} \mathbf{R}^{-1} \mathbf{B}^{T} \mathbf{P}_{c}+\mathbf{M}^{T} \mathbf{Q M}=\mathbf{0} .
$$

Similarly the Kalman filter gain now is given by

$$
\mathbf{K}_{f}=\mathbf{P}_{f} \mathbf{C}^{T} \mathbf{V}^{-1}
$$

where $\mathbf{V}$ is real symmetric positive definite matrix and $\mathbf{P}_{f}$ is the symmetrical solution matrix of the KF Riccati equation given by

$$
\mathbf{P}_{f} \mathbf{A}^{T}+\mathbf{A} \mathbf{P}_{f}-\mathbf{P}_{f} \mathbf{C}^{T} \mathbf{V}^{-1} \mathbf{C} \mathbf{P}_{f}+\Gamma^{T} \mathbf{W} \Gamma=0,
$$

where $\mathbf{P}_{c}=\mathbf{P}_{c}^{T} \geq 0$ and $\mathbf{P}_{f}=\mathbf{P}_{f}^{T} \geq 0$ and $\mathbf{Q}, \mathbf{R}, \mathbf{V}$, and $\mathbf{W}$ are weight matrices which can be regarded as setting parameters (tuning) that must be manipulated until they find one acceptable response to the system.

A necessary and sufficient condition to guarantee the existence of the $\mathbf{K}_{c}$ and $\mathbf{K}_{f}$ is if the system is completely controllable and observable. The LQG method is more realistic than the LQR method, since the states are not always available to be feedback or need to be measurable with the aid of sensors. The inclusion of the noise in the model which represents imperfections of the system is also one advantage of the LQG method.

\section{Simulations and Results}

The first simulation is the design of the control law using the LQR and LQG control theories, for the spacecraft with a partially filled tank, to account for the sloshing dynamics by the mechanically analog pendulum type. The pendulum physical parameters used in the simulations are as follows: $m=600 \mathrm{~kg}, m_{f}=100 \mathrm{~kg}, I=720 \mathrm{~kg} / \mathrm{m}^{2}, I_{f}=90 \mathrm{~kg} / \mathrm{m}^{2}$, $a=0.3 \mathrm{~m}, b=0.3 \mathrm{~m}, F=500 \mathrm{~N}$, and $\varepsilon=0.19 \mathrm{~kg} \mathrm{~m}^{2} / \mathrm{s}$. The equations of motion that describe the dynamics of the system are given by (13) which need to be put in state space form. These equations describe the angular displacement of the spacecraft and the angular displacement of the pendulum and the initial conditions used are $\theta=2^{\circ}, \dot{\theta}=0.57^{\circ} / \mathrm{s}, \psi=1^{\circ}$, and $\dot{\psi}=0^{\circ}$.

Figures 2 and 3 show that the LQR control law performance is better than the LQG and the reason is because the first one considers that the sloshing variables are available to be feedback which is not true.

The performance of the LQG control law is damaged because the sloshing motion is controlled indirectly and 




(a)

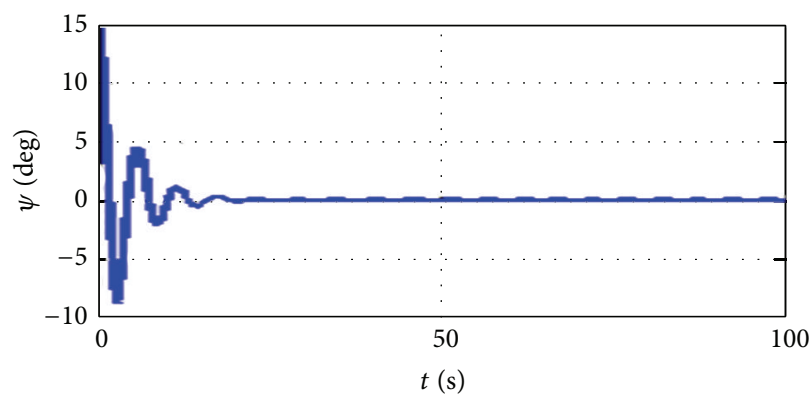

(c)

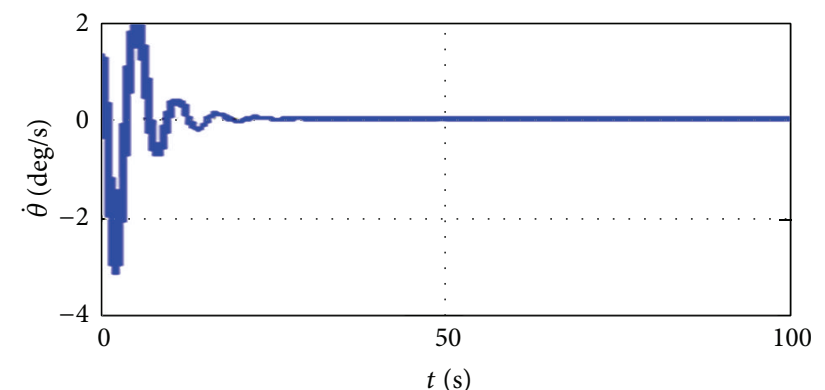

(b)

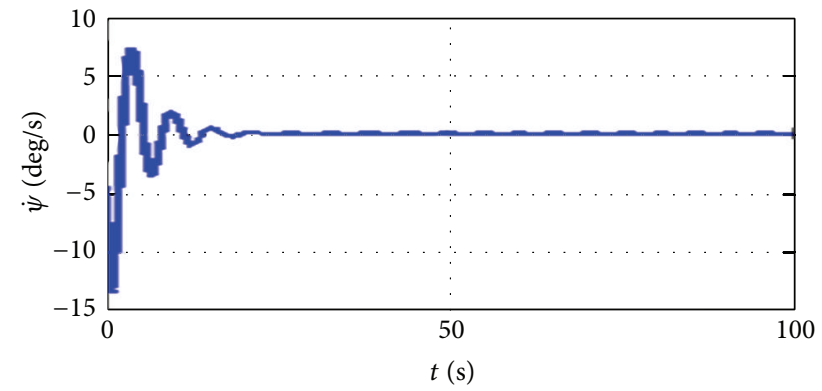

(d)

FIgURe 2: Performance of the LQR control law.

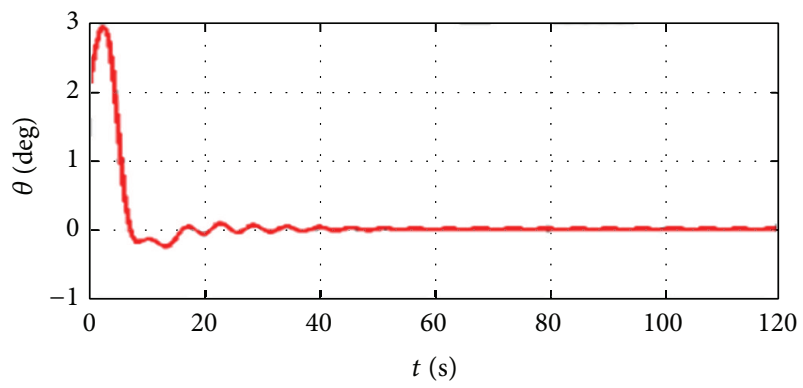

— LQG

(a)

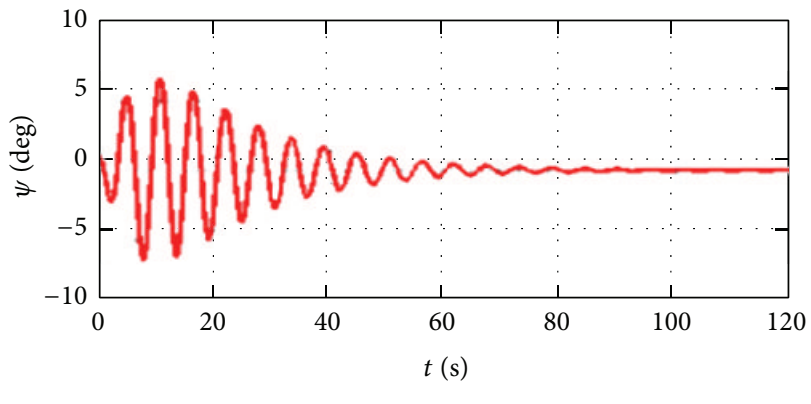

— LQG

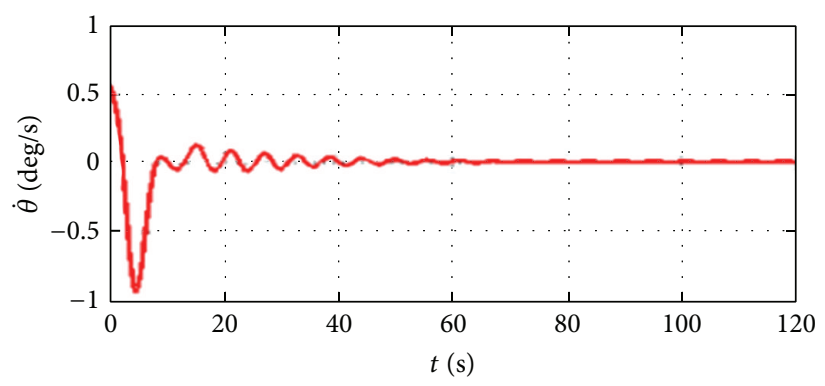

— LQG

(b)

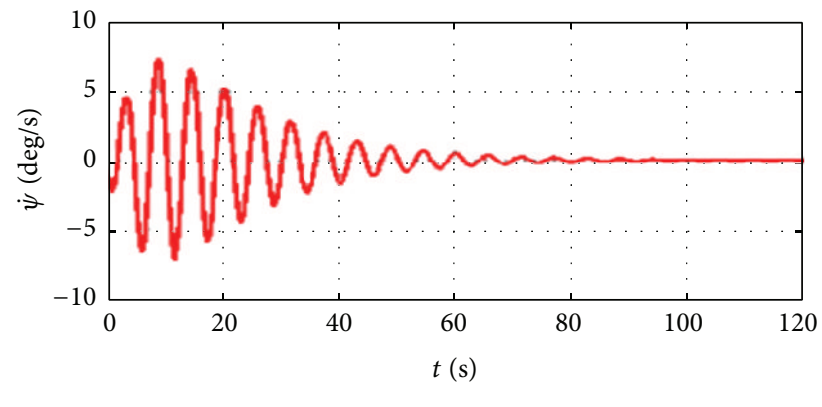

— LQG

(c)

(d)

FIGURE 3: Performance of the LQG control law. 


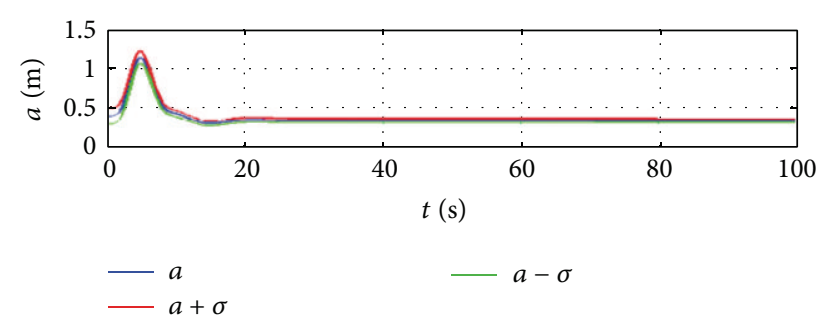

(a)

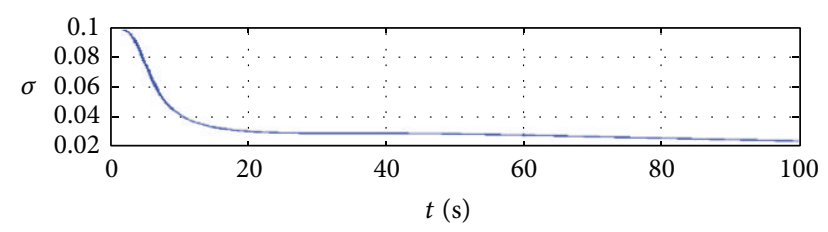

(b)

Figure 4: The estimation of the rod length.

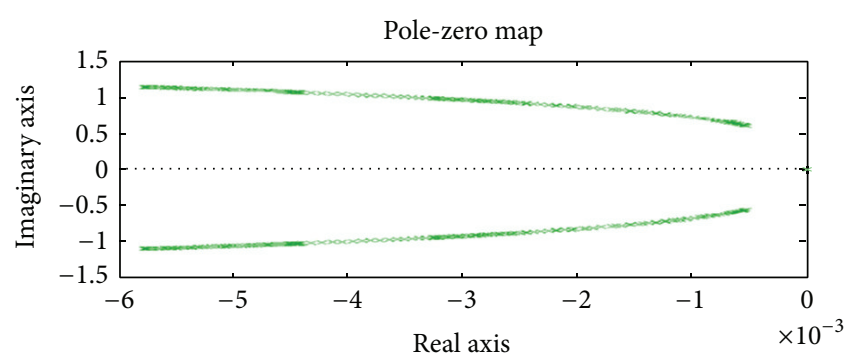

FIGURE 5: Poles position during the estimation.

the sloshing state variables are not available to be feedback and they need to be estimated by the Kalman filter.

Now one uses the Kalman filter to estimate the rod length of the pendulum using a database containing the time evolution of the variables $\theta$ and $\dot{\theta}$. The rod length $a$ is now considered as state variable so one has a system with five state variables which can be estimated. Using the same control LQR law, it is possible to analyze the control system performance during the estimation of the rod length. Besides, one inserts a white noise in the measurements $\theta$ and $\dot{\theta}$, given by the noise process $v_{k}=N(0,0.001)$.

Figure 4 shows that the estimated value of the rod length is $a=0.33 \mathrm{~m}$, remembering that the "actual" value of the rod is $0.3 \mathrm{~m}$. Figure 5 shows that plant of the system depends on the estimation of the rod, since at the time that the rod length is varying the position of the poles also moves.

Figures 6 and 7 show the LQR and the LQG controllers during the rod length estimation. One observes that the system is controlled in less than approximately 15 seconds and that the slosh motion is more oscillatory with the LQG controller. This is because the sloshing motion is controlled indirectly and the sloshing state variables are being estimated by the Kalman filter.

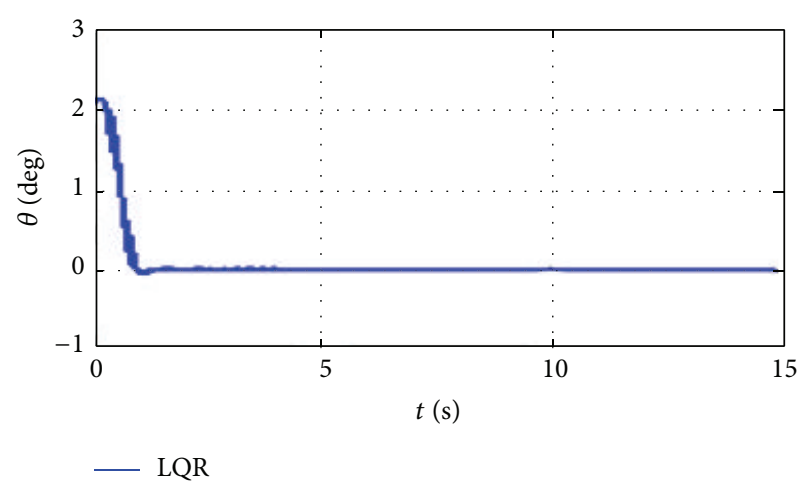

(a)

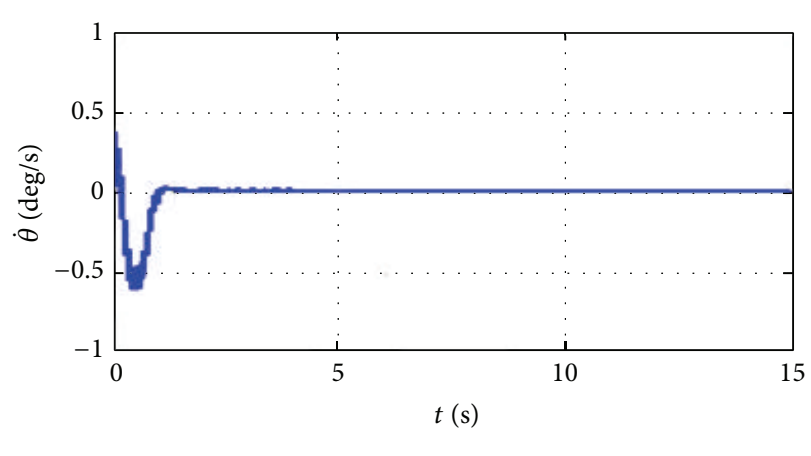

- LQR

(b)



(c)

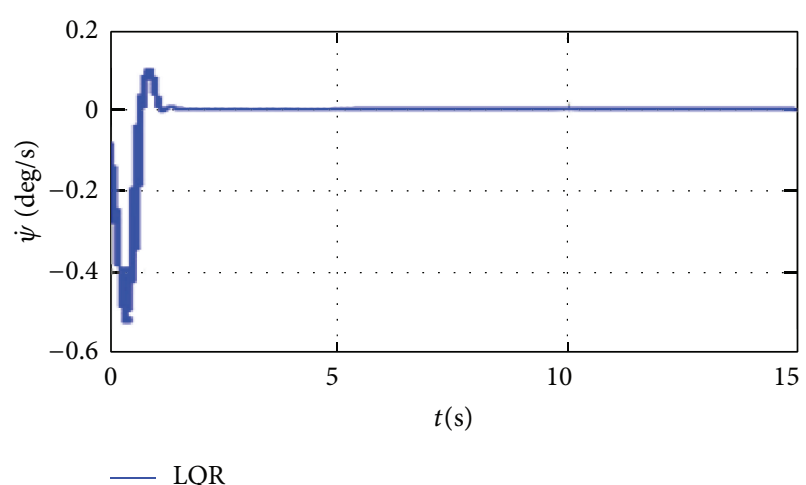

(d)

FIGURE 6: LQR controller during the rod estimation. 


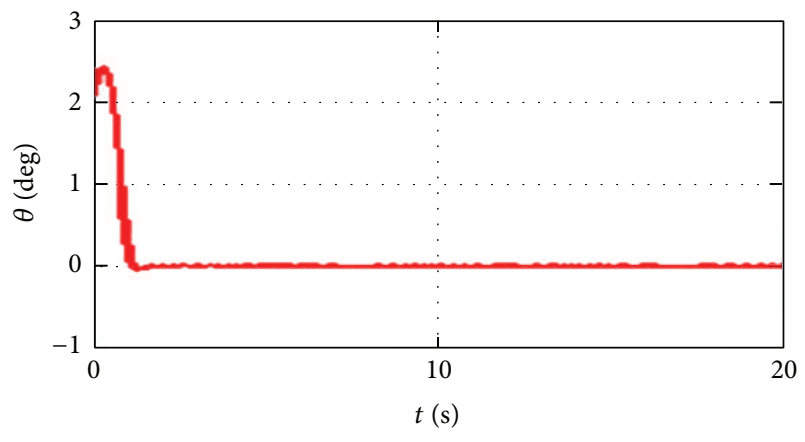

— LQG

(a)

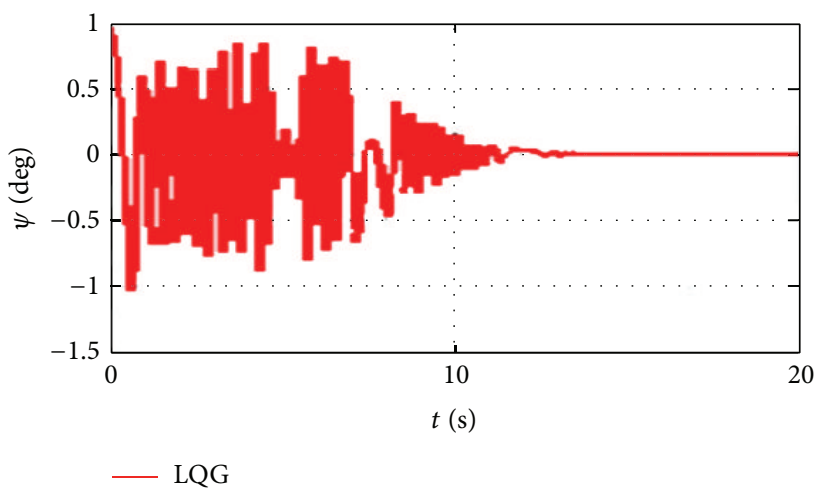

(c)

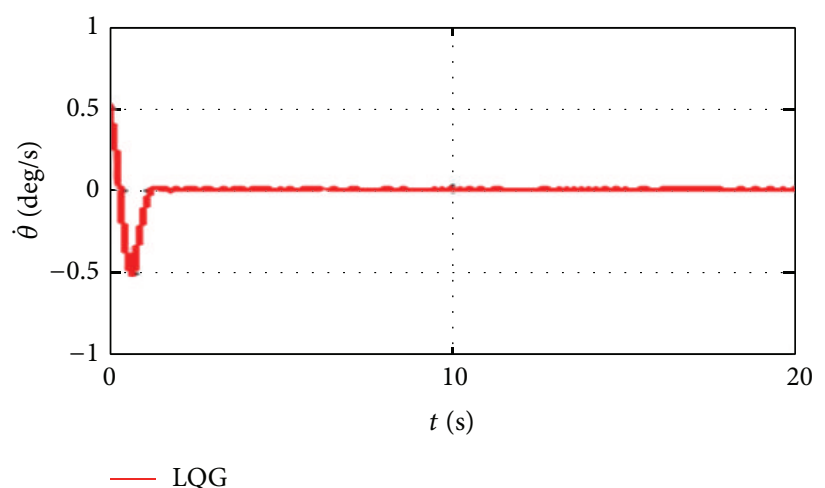

(b)



(d)

FIGURE 7: LQG controller during the rod estimation.

\section{Conclusions}

In this paper one described briefly the concepts of the sloshing phenomenon which is associated with the dynamics of a liquid moving into at partially fills reservoir. Deriving the equation of motion of a spacecraft with liquid inside the sloshing phenomenon is represented by its mechanical analog of a pendulum type. One uses the Kalman filter technique to estimate the pendulum length. One shows that the performance of the LQR control is better than the LQG control during the estimation process. The reason the LQG control degraded is because the sloshing states need to be estimated by the filter. This degradation was expected, because as it has been demonstrated by [15] the introduction of the Kalman filter degrades the stability margin of the LQG. One way to improve the performance of LQG is to make the dynamics of the filter faster than the dynamics of LQR. However, this must be done carefully since this time will depend on the on-board computer capacity. These results show that control of spacecraft with sloshing and flexibility is not an easy task and needs to be investigated better. One has also observed that the estimation of the rod length alters the plant of the system causing the poles to walk to and from the imaginary system, leaving in the end the plant more stable.

\section{Conflict of Interests}

The authors declare that there is no conflict of interests regarding the publication of this paper.

\section{References}

[1] R. Barron and S. W. R. Chng, "Dynamic analysis and measurement of sloshing of fluid in containers," Journal of Dynamic Systems, Measurement and Control, vol. 111, no. 1, pp. 83-90, 1989.

[2] S. K. Chakrabarti, "Internal waves in a large offshore storage tank," Journal of Energy Resources Technology, vol. 115, no. 2, pp. 133-141, 1993.

[3] D. E. Hill and J. R. Baumgarten, "Control of spin-stabilized spacecraft with sloshing fluid stores," Journal of Dynamic Systems, Measurement and Control, vol. 114, no. 4, pp. 728-731, 1992.

[4] A. P. C. Lui and J. Y. K. Lou, "Dynamic coupling of a liquid-tank system under transient excitations," Ocean Engineering, vol. 17, no. 3, pp. 263-277, 1990.

[5] B. N. Agrawal, "Dynamic characteristics of liquid motion in partially filled tanks of a spinning spacecraft," Journal of Guidance, Control, and Dynamics, vol. 16, no. 4, pp. 636-640, 1993. 
[6] M. J. Sidi, Spacecraft Dynamics and Control, Cambridge University Press, New York, NY, USA, 1997.

[7] L. C. G. Souza, "Robust controllers design for flexible space system using a combination of LQG/LTR and PRLQG methods," in Dynamics and Control of Structure in Space III, C. L. Kirk and D. J. Inman, Eds., pp. 151-166, Computational Mechanics, 1996.

[8] G. T. Conti and L. C. G. Souza, "Satellite attitude control system simulator," Shock and Vibration, vol. 15, no. 3-4, pp. 395-402, 2008.

[9] L. C. G. Souza and R. G. Gonzales, "Satellite simulator control system design using SDRE method," Journal of Mechanics Engineering and Automation, vol. 1, pp. 123-132, 2011.

[10] L. C. G. Souza, "Inertia parameters estimation of a satellite simulator," in Advances in Astronautical Sciences, vol. 1304, pp. 969-979, 2008.

[11] R. A. Ibrahim, Liquid Sloshing Dynamics, Theory and Applications, Cambridge University Press, New York, NY, USA, 2005.

[12] A. G. Souza, Study of the effects of liquid movement and flexibility in performance and robustness of the attitude control system of an artificial satellite [Master of Space Mechanics and Control], National Institute for Space Research (INPE), São José dos Campos, Brazil, 2013, http://urlib.net/8JMKD3MGP7W/ 3DG55TB.

[13] D. E. Kirk, Optimal Control Theory: An Introduction, Dover, Mineola, NY, USA, 1998.

[14] L. C. G. de Souza and X. C. Méndez Cubillos, "Using of Hinfinity control method in attitude control system of rigidflexible satellite," Mathematical Problems in Engineering, vol. 2009, Article ID 173145, 9 pages, 2009.

[15] J. C. Doyle, "Guaranteed margins for LQG regulator," IEEE Transactions on Automatic Control, vol. 23, no. 4, pp. 756-757, 1978. 

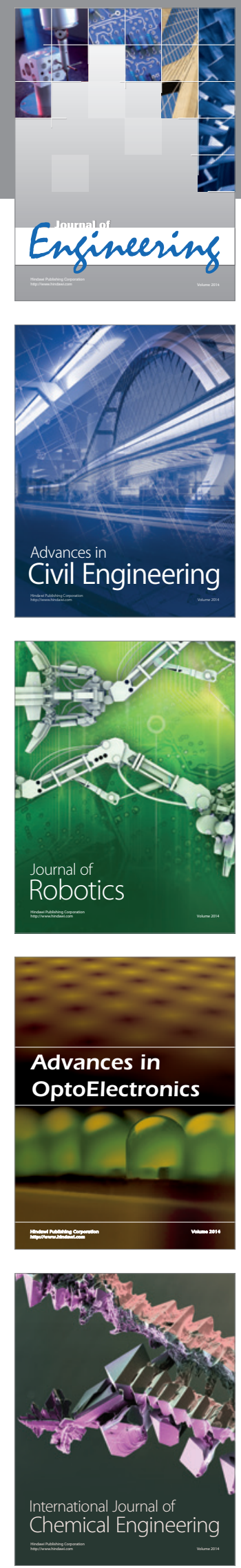

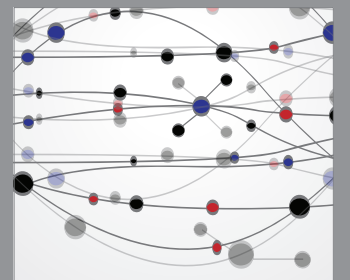

The Scientific World Journal
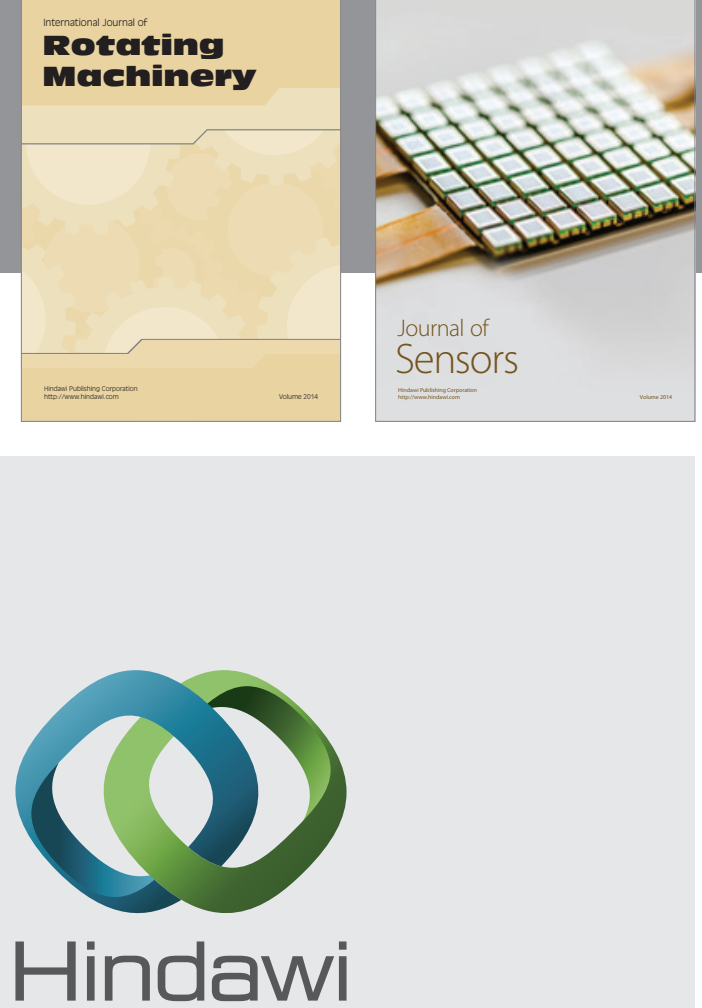

Submit your manuscripts at http://www.hindawi.com
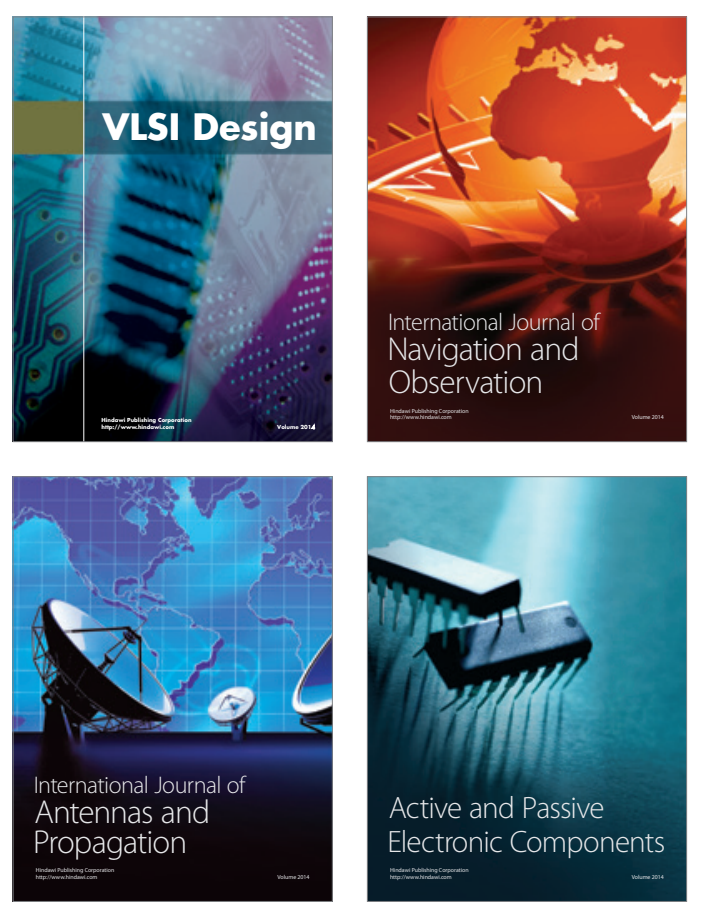
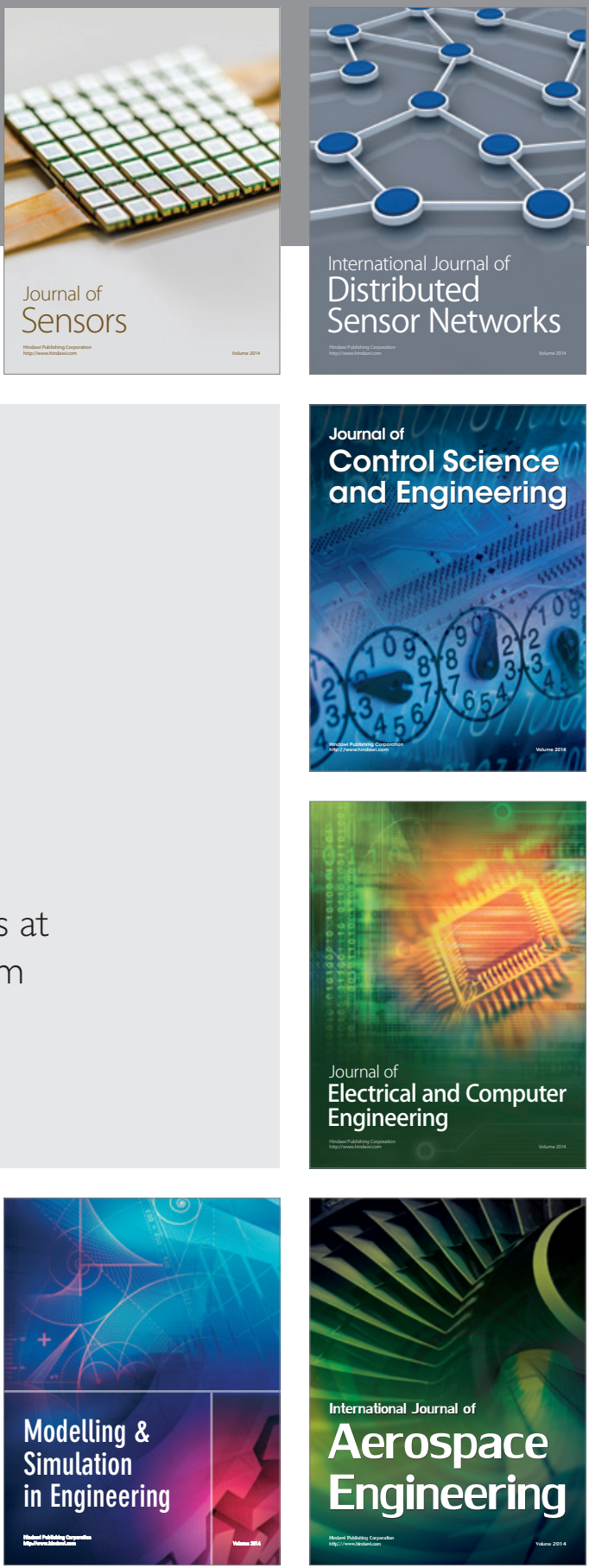

Journal of

Control Science

and Engineering
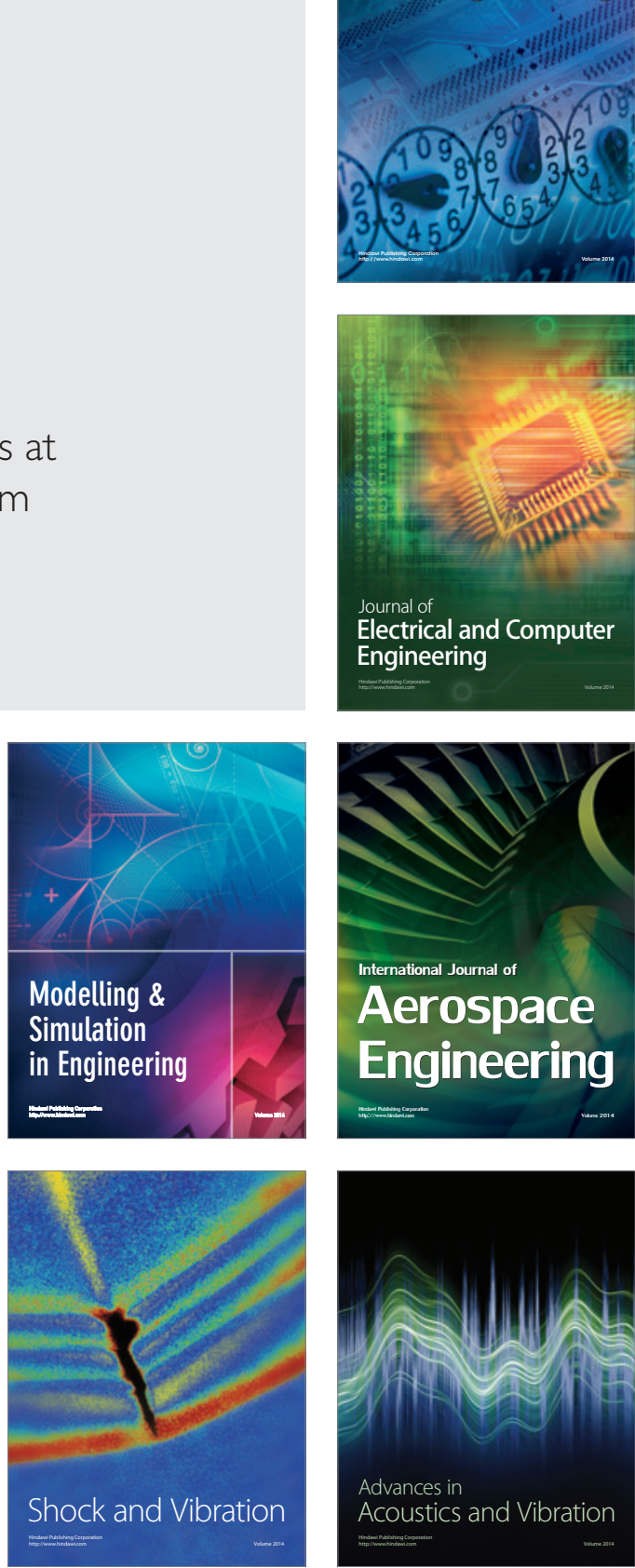